Check for updates

Richmond, VA

carrierobin@gmail.com Follow Carrie on Twitter @edbites

Cite this as: BMJ 2020;371:m4751

http://dx.doi.org/10.1136/bmj.m4751

Published: 17 December 2020
DRUG MISUSE

\section{The US covid pandemic has a sinister shadow-drug overdoses}

\section{The covid-19 pandemic has worsened the US opioid crisis, and policy changes have not done enough to help, reports Carrie Arnold}

\section{Carrie Arnold independent public health journalist}

As the United States began shuttering non-essential businesses and closing schools to slow the spread of covid-19 in mid-March, Richard Jorgensen braced himself for the possibility of mass deaths. As the coroner for DuPage County in Illinois, just west of Chicago, Jorgensen knew that his job would be to investigate deaths from the burgeoning pandemic. By the end of March, he was indeed seeing a surge in deaths. But they weren't from the coronavirus. They were from drug overdoses.

Jorgensen was no stranger to deaths from overdose. In the summer of 2013, not long after he became coroner, his office registered 18 such deaths in 18 days. "For me, that was just shocking," he recalls.

This year, the social isolation of the lockdown was dovetailed with financial chaos as many low wage workers found themselves jobless, fueling a surge of deaths by suicide and opiate overdose. Jorgensen ultimately logged 20 deadly overdoses in three weeks in April to May-by far the highest he'd seen since 2013.

"We had a number of days where we had two or three overdoses in a day," he says. "We're still not anywhere close to being in control of this thing."

This rise wasn't unique to DuPage County, either. Throughout the US, a shadow pandemic of drug overdoses has emerged in the wake of the coronavirus. Preliminary data from October 2020, published by the National Center for Health Statistics at the US Centers for Disease Control and Prevention, ${ }^{1}$ showed that over 19000 Americans had died as the result of an overdose in the first three months of 2020, just weeks into the pandemic-an increase of $16 \%$ since 2019. The uptick came just as some parts of the country were finally seeing a hard fought downturn in overdoses and deaths.

\section{Progress eaten away}

Preliminary data from other sources show that the US is on track to record an all time peak of overdose deaths in 2020. The University of Michigan's System for Opioid Overdose Surveillance found that deaths from suspected overdose in the state from 1 March to 16 September were $15 \%$ higher than the same period in 2019. ${ }^{2}$ Seven states showed increases greater than $25 \%$.

Reports from the Overdose Detection Mapping Application Program, ${ }^{3}$ which provides overdose data in near real time to public safety and public health organizations in various states, show a similar increase during 2020. Paramedics are also reporting more naloxone use to reverse opioid overdoses-a sign that overdoses in general are on the rise, not just deadly ones.

"Right now, we are seeing the epidemic within the pandemic, and it's really continuing to rise rather than stay the same," says Beth Connolly, project director of the Substance Use Prevention and Treatment Initiative at the Pew Charitable Trusts. "Some states had made really good progress prior to the pandemic, and now some of this progress is being eaten away."

The lockdown created the perfect storm for substance misuse and overdose, says Courtney Hunter, vice president for state policy at Shatterproof-a non-profit group working to improve addiction treatment policies-because it disrupted routines and in-person support services. Economic and coronavirus worries triggered relapses even in people who had been sober for years. Those who did start using again often turned to multiple substances simultaneously, including opioids, benzodiazepines, and alcohol. And social distancing guidelines made people more likely to get high alone, away from those who could call emergency services or administer naloxone.

Patrice A Harris, past president of the American Medical Association and chair of its Opioid Task Force, said in a statement, "The covid-19 pandemic has collided with the ongoing drug overdose epidemic, making it more difficult for patients with a substance use disorder to access evidence based care; more challenging for patients with pain; and more difficult for harm reduction organizations to distribute naloxone or sterile needles and syringes."

Harris called for policy to require health insurers to stop limiting access to medicines and pain care. If insurers aren't required to provide better addiction care, she warned, "more Americans will continue to suffer and die."

\section{Loss of contact}

The addiction specialist Peter Grinspoon had a front row seat for the early chaos at his bustling clinic in Chelsea, Massachusetts, a gritty inner suburb of Boston. As the coronavirus began to tear through the area in late February, public health officials started issuing social distancing guidelines and orders to stay home.

Many of Grinspoon's patients were minimum wage healthcare workers or janitors, who were classified as essential workers and had to go to work. And many relied on methadone, buprenorphine, or the 
buprenorphine-naloxone combination Suboxone as part of their recovery from opioid addiction, all of which required in-person clinic visits. Methadone users in particular needed to line up daily to receive their small cups of the fuchsia liquid. That meant large groups of people standing in close proximity, many of whom were highly exposed to the coronavirus and had weakened immune systems. "This is a recipe for disaster," says Grinspoon.

Harm reduction clinics that provided needle exchanges and supervised drug use centers also closed or reduced their hours, especially in the early days of the pandemic. These facilities not only provided clean needles but also supplied naloxone and fentanyl test strips, which helped prevent overdoses and deaths, and referrals to rehab when their clients felt ready.

On 16 March the Substance Abuse and Mental Health Services Administration eased regulation of the three drugs used to treat opioid addiction, for the duration of the ongoing public health emergency. ${ }^{4}$ This allowed patients to receive several days' or weeks' worth of methadone and get prescriptions and refills through telemedicine. Although Connolly praised the move, saying that this was something whose time had come, not all methadone clinics were set up to make the switch immediately, and the changes were voluntary-some clinics didn't switch, she says.

Treatment centers that remained open were often overwhelmed by people seeking help. US law requires special training and certification for physicians prescribing buprenorphine for addiction-though not, Connolly says wryly, for doctors prescribing the same medicine to treat pain-so it was almost impossible for other physicians to step in and pick up the slack.

The shift in these rules undoubtedly saved some lives by preventing deadly relapses and coronavirus spread, says Grinspoon, but he believes that the reduction in face-to-face care and interactions with fellow patients also did harm. Many people working to recover from substance misuse also attend Alcoholics Anonymous, Narcotics Anonymous, or other support groups that have historically met in person. Some have been attending the same meeting in the same location with the same people for decades. Younger attendees and those newer to recovery have been better able to make the shift to virtual support, but the old hands have had a harder time adjusting, says Grinspoon.

\section{New policy needed}

From a policy perspective, Hunter says that the switch to virtual recovery services needs to consider people who aren't digital natives. Video chat doesn't work for people without smartphones, she says, and keeping enough minutes on a cell phone plan to contact health providers or other support can be a challenge for people who struggle financially. Where telemedicine is the only option, it would be a huge step forward to make these tools accessible to everyone.

Even under the best circumstances, however, addiction is a disease prone to relapse. An estimated $40-60 \%$ of people who misuse drugs will return to using them at some point during recovery-and it's this time that leaves them most vulnerable to overdose, since their tolerance has dropped, often dramatically, during their period of abstinence.

This fact was common knowledge in addiction medicine even in pre-covid times, says Grinspoon. Financial crises and natural disasters often cause an uptick in people relapsing and turning to drugs and alcohol to cope, so he wasn't surprised when he heard preliminary reports that more people were using and overdosing. "In a perfect world, we'd all meditate, exercise and, you know, chant or whatever-but in reality people reach for drugs and alcohol," he says. "It's hard enough to make it by day to day when you're recovering from a chronic disease that's stigmatized."

Drug overdoses during the covid pandemic have been especially deadly because people who might otherwise have used in a group, or with others at home or nearby, have more frequently been using alone. This means that no one is around them to use naloxone and call for an ambulance if they begin overdosing, and overdoses that might have been otherwise survivable become deadly.

The surge in deaths from overdose in the wake of covid-19 highlights just how much more work is ahead for the US in tackling the opioid crisis, says Courtney Hunter. To do that, physicians and policy makers need to move addiction treatment out of its silo, she explains-and, despite the pandemic, now is as good a time as any. "We have to really train people to think of addiction just like any other disease," she says. "And by doing that, we can hopefully integrate it into the medical mainstream."

Joe Biden's incoming administration may bring a new emphasis to the US's treatment and prevention programs-his son Hunter has struggled with substance abuse, and Biden has talked about various plans to combat the opioid epidemic. He has promised \$125bn (£93.6bn; €103.1bn) for treatment programs, but observers say that getting the plan through a divided Congress could be tough. ${ }^{5}$

Competing interests: I have read and understood BMJ policy on declaration of interests and have no relevant interests to declare.

Provenance and peer review: Commissioned, not peer reviewed.

Centers for Disease Control and Prevention, National Center for Health Statistics. Percent of drug overdose deaths by quarter and demographic characteristics: United States 2018-Q1 through 2020-Q1. https://www.cdc.gov/nchs/data/health_policy/Provisional-Drug-Overdose-Deaths-byQuarter-Demographic-Characteristics-Q1-2020.pdf.

2 University of Michigan Injury Prevention Center. System for opioid overdose surveillance (SOS). 2020. http://injurycenter.umich.edu/opioid-overdose/opioid-surveillance/.

3 Overdose Detection Mapping Application Program. User stories: ODMAP in action. http://www.odmap.org/.

4 Substance Abuse and Mental Health Services Administration. Opioid Treatment Program (OTP) Guidance. Updated 19 Mar 2020. https://www.samhsa.gov/sites/default/files/otp-guidance20200316.pdf.

5 Goldberg D, Ehley B. Biden's other health crisis: a resurgent drug epidemic. Politico 2020 Nov 28. https://www.politico.com/news/2020/11/28/bidens-other-health-crisis-a-resurgent-drugepidemic-440771. 